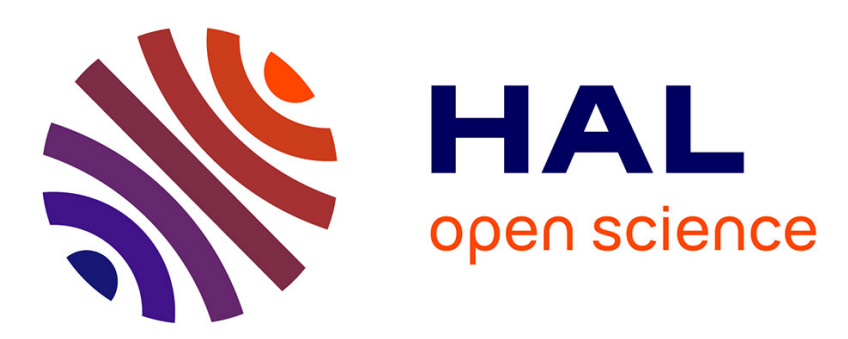

\title{
Orientations actuelles de la sélection de variétés résistantes aux maladies chez les plantes maraîchères
}

\author{
Michel Clerjeau, H. Laterrot, Hervé Lecoq, Michel Pitrat
}

\section{To cite this version:}

Michel Clerjeau, H. Laterrot, Hervé Lecoq, Michel Pitrat. Orientations actuelles de la sélection de variétés résistantes aux maladies chez les plantes maraîchères. Agronomie, 1981, 1 (1), pp.41-48. 10.1051/agro:19810106 . hal-02728543

\section{HAL Id: hal-02728543 \\ https://hal.inrae.fr/hal-02728543}

Submitted on 2 Jun 2020

HAL is a multi-disciplinary open access archive for the deposit and dissemination of scientific research documents, whether they are published or not. The documents may come from teaching and research institutions in France or abroad, or from public or private research centers.
L'archive ouverte pluridisciplinaire HAL, est destinée au dépôt et à la diffusion de documents scientifiques de niveau recherche, publiés ou non, émanant des établissements d'enseignement et de recherche français ou étrangers, des laboratoires publics ou privés. 


\title{
Orientations actuelles de la sélection de variétés résistantes aux maladies chez les plantes
} maraîchères

\author{
Michel CLERJEAU $\left({ }^{*}\right)\left({ }^{1}\right)$, Henri LATERROT $\left({ }^{* *}\right)$, Hervé LECOQ $\left({ }^{*}\right)$, Michel PITRAT $\left({ }^{* *}\right)$ \\ (*) Station de Pathologie végétale, $\left({ }^{* *}\right)$ Station d'Amélioration des Plantes maraîchères, \\ Centre de Recherches d'Avignon I.N.R.A., 84140 Montfavet
}

RESUME

Résistance absolue, Résistance partielle, Cumul de gènes, Lutte intégrée, Durabilité de la résistance.
L'introduction de caractères de résistance aux maladies chez les plantes maraîchères a surtout été fondée, dans le passé, sur l'utilisation de gènes majeurs de résistance absolue. La sélection prenait alors en compte, essentiellement, le niveau de la résistance. Les diverses situations auxquelles elle a abouti sont examinées (succès et échecs).

Les tendances actuelles des sélectionneurs ont, par rapport au passé, l'originalité de prendre davantage en compte le problème de la durabilité des résistances et l'intérêt des résistances partielles (moindre sensibilité). Les travaux effectués au Centre I.N.R.A. d'Avignon-Montfavet, par les sélectionneurs et les pathologistes. servent à illustrer ces deux notions :

- La mise en évidence de "gènes forts " de résistance et la mise au point de variétés possédant plusieurs mécanismes de résistance fondamentalement différents, afin d'améliorer la durabilité des résistances, sont des aspects particulièrement développés (utilisation de résistances polygéniques, cumul de gènes induisant des mécanismes de défense différénts).

- Le problème de l'exploitation des caractères de résistance partielle est principalement abordé sous l'angle d'une intégration de la résistance variétale à d'autres méthodes de lutte (chimiques ou biologiques).

\section{ABSTRACT}

Absolute resistance. Partial resistance. Adding of genes. Integrated control, Resistance durability.

\section{Current trends in the breeding of resistant vegetable varieties}

The introduction of disease resistance characters into vegetable varieties during the past has been done using absolute resistance major genes. Until recently, resistance level was taken as of principal importance. The various sịtuations resulting from such breeding strategy (successes and failures) are discussed.

The trends of breeding work have changed during the last few years : the problem of resistance durability and the interest of partial resistances (lower susceptibility) are now being given greater consideration. Work carried out by breeders and plant pathologists in the I.N.R.A. research center of Montfavet-Avignon (France) are discussed here to illustrate these two features :

- The assessment of the strength of resistance genes (with special reference to Tomato resistance to Cladosporium fulvum, Muskmelon resistance to CMV and Pepper resistance to PVY) and the development of varieties possessing several different resistance mechanisms will lead to the development of varieties with more durable resistance because they take into account the aggressiveness of plant pathogens. Two ways are examined and discussed by which to accumulate several resistance factors : the use of polygenic resistance (ex.: Pepper-Phytophthora capsici), which does not exclude a possible parasite adaptation to some components of this type of resistance, and the addition of various known major or minor genes governing different resistance mechanisms (ex.: Muskmelon-Fusarium oxysporum f. sp. melonis ; Tomato-TMV; Muskmelon-CMV).

- The problem of the use of field genes which induce partial resistance, or only lower susceptibility, is approached here from the aspect of integrated disease control. It has been shown for $P$. capsicion Pepper and CMV on Muskmelon that some weak fungicide drenches or some cultural practices can enhance the varietal resistance efficiency by decreasing the amount of inoculum.

\section{INTRODUCTION}

L'introduction de caractères de résistance aux maladies chez les plantes légumières concerne principalement les espèces les plus répandues en cultures intensives (Tomate,

(1) Nouvelle adresse : Station de Pathologie végétale, Centre de Recherches de Bordeaux, I.N.R.A., Pont-de-la-Maye, 33140 Villenave d'Ornon.
Laitue, Melon, Concombre) mais aussi quelques espèces de grandes cultures (Pois, Haricot) et de plus rares productions secondaires (Poivron, Aubergine, Courgette). Dans ce domaine de l'amélioration des plantes, des contraintes spécifiques pèsent sur le sélectionneur. Celles-ci sont liées :

- à la très grande diversité des espèces végétales concernées et à leurs types de production ainsi qu'au nombre important de maladies potentiellement graves qui peuvent 
les atteindre. Il en résulte que le nombre de résistances que les sélectionneurs de plantes maraîchères sont amenés à manipuler est considérable ;

- aux évolutions permanentes et rapides des exigences du marché, ainsi que des techniques culturales, qui remettent en cause fréquemment les choix variétaux ;

- aux exigences des producteurs pour qui la résistance génétique à une maladie n'est qu'exceptionnellement un élément de choix variétal déterminant si une méthode de lutte chimique permet de protéger efficacement une variété sensible de grand intérêt agronomique ou économique.

Cet ensemble de facteurs limite incontestablement la recherche de solutions génétiques à long terme. Il explique partiellement les choix stratégiques de sélection du passé et doit éclairer les choix du futur. Le développement qui suit se propose précisément d'indiquer dans quel sens ont évolué et s'orientent actuellement les objectifs et méthodologies de la sélection chez les plantes légumières. Les exemples choisis sont essentiellement empruntés aux travaux réalisés dans le Centre I.N.R.A. d'AvignonMontfavet.

\section{LA SELLECTION DANS LE PASSÉ}

Jusqu'à ces dernières années, la création par les sélectionneurs de variétés résistantes chez les plantes légumières a surtout reposé sur l'introduction de gènes dominants de résistance absolue. La notion d'efficacité de la résistance étant alors, en grande partie, assimilée à celle de niveau de résistance, seuls ces gènes semblaient adaptés aux objectifs de sélection.

Du fait de la faillite de certains gènes qui ont amené les sélectionneurs à en rechercher de nouveaux. surmontés à leur tour dès leur utilisation (ex. : résistance à Bremia lactucae Regel chez la Laitue), on a souvent fait le reproche à cette sélection d'être aveugle ou suicidaire.

L'objectivité impose de dire que, dans la majorité des cas, les géniteurs de résistance ou leurs descendants étitient généralement expérimentés durant plusieurs saisons, dans diverses régions, avant le stade de création variétale proprement dit. C'était un moyen non négligeable d'éprouver la qualité des gènes retenus.

Cette sélection qui, aujourd'hui encore, reste la plus fréquente, a abouti à trois types de situation:

- Des cas d'échecs graves vis-à-vis de certaines maladies, qui se sont caractérisés par l'apparition de nouveaux pathotypes virulents, rapidement après l'utilisation des gènes de résistance (ex.: 8 gènes connus de résistance à $B$. lactucae surmontés chez la Laitue ; 5 gènes de résistance à Cladosporium fulvum Cooke surmontés chez la Tomate). On peut qualifier ces gènes de gènes faibles de résistance verticale au sens de VAN DER PLANK (1968). Ces cas souvent cités en exemple, sont en fait, relativement peu fréquents.

- Des cas de brillants succès où les gènes utilisés depuis de nombreuses années n'ont encore jamais été surmontés. Les exemples de ce type sont au moins aussi nombreux que les précédents : gène $\mathrm{Ccu}$ de résistance à Cladosporium cucumerinum Ellis et Arth. chez le Concombre ; gène Sm de résistance à Stemphylium solani Weber chez la Tomate ; gène $\mathrm{Tm}-2^{2}$ de résistance au virus de la Mosaïque du Tabac (TMV) chez la Tomate également. On peut alors parler de gènes forts.

- Des cas intermédiaires où des pathotypes adaptés aux gènes de résistance sont apparus, mais dont l'agressivité est faible ou dont l'extension géographique reste limitée. Dans ces conditions, la portée agronomique et économique de l'adaptation est réduite. Comme exemples de résistances de ce type, nous pouvons citer celle du Melon à Fusarium oxysporum Schlecht f. sp. melonis due aux gènes Fom-1 et Fom-2 (races adaptées peu agressives), celle du Haricot à Colletotrichum lindemuthianum (Sacc. et Magn.) Briosi et Cav. due au gène Are ou du Piment au TMV due au gène $\mathrm{Li}$ (races adaptées peu répandues pour ces derniers cas).

En conclusion, les échecs imputables à l'utilisation de certains gènes majeurs ne doivent pas masquer l'intérêt que leur usage peut continuer à présenter et ne semblent pas justifier la condamnation sans appel que certains leur adressent. En revanche, ces échecs ont fait prendre conscience d'une dimension de la résistance, peu perçue à l'origine des programmes, celle de la durabilité. Cette notion nouvelle conditionne largement les stratégies actuelles de sélection.

\section{TENDANCES DE LA SILECTION D'AUJOURD'HUI}

Afin de mieux comprendre la démarche des sélectionneurs, il est indispensable d'indiquer quels sont les objectifs de ceux-ci en matière de niveau et de durabilité de la résistance.

\section{1) Le problème du niveau de la résistance}

La recherche de très hauts niveaux de résistance n'est plus aujourd'hui un objectif fondamental. L'essentiel paraît être d'introduire des niveaux suffisants pour réduire les épidémies à un seuil acceptable. Les sélectionneurs négligent donc de moins en moins les résistances partielles ou conditionnelles. De telles résistances sont souvent oligo ou polygéniques mais peuvent être monogéniques. C'est le cas du gène $\mathrm{Ph}-2$ de résistance partielle au mildiou chez la Tomate. Pour trier les éventuels géniteurs, les méthodes d'inoculations artificielles doivent être moins drastiques que celles qui permettent de cribler les résistances absolues. Il s'agit souvent de méthodes d'appréciation quantitatives utilisant soit des souches d'agents pathogènes peu agressives, soit de faibles doses d'inoculum, mises en œuvre dans des conditions de milieu particulières (CLERJEAU et al., 1979).

\section{2) Le problème de la durabilité}

La rapidité d'adaptation d'un parasite donné à un génotype de plante résistante est surtout fonction de la nature des gènes de résistance qui lui sont opposés, ainsi que de la stratégie d'utilisation de ces gènes dans l'espace et dans le temps. La durabilité d'une résistance repose donc sur le choix des gènes et les modalités de leur utilisation.

- Le choix des gènes est un problème qui se pose essentiellement aux sélectionneurs. Il doit s'opérer grâce à une meilleure appréciation a priori de la «force» de ces gènes (sensu VAN DER PLANK). Cela implique notamment l'acquisition de données sur la variabilité génétique du pouvoir pathogène des parasites ou l'aptitude à la compétition des éventuels pathotypes adaptés aux gènes de résistance utilisables.

- La stratégie d'utilisation des gènes, quant à elle, à la différence de leur choix, n'est pas sous la seule dépendance d'un progrès de connaissances mais aussi d'aspects qui touchent les structures socio-économiques. Il importe en 
effet que les solutions proposées soient compatibles avec les exigences des structures ou des systèmes agraires en place. Or, les cultures maraîchères ne présentent pas une valeur stratégique nationale suffisante pour donner à l'avis des chercheurs un poids déterminant. Dans le domaine de la sélection des plantes légumières, il faut donc concilier le scientifiquement souhaitable et l'économiquement possible. Les solutions nouvelles devant éviter l'utopie, nous exposerons ici quelques-unes des voies qui paraissent les plus prometteuses pour y parvenir.

\section{VOIES DE RECHERCHES ET OBJECTIFS DE SÉLECTION PROPRES À AMÉLIORER LA DURABI- LITÉ DES RESISTANCES}

\section{1) Mise en évidence de gènes "forts " de résistance}

Lorsque le sélectionneur dispose de plusieurs géniteurs de résistance à un agent pathogène (qu'elle soit mono ou polygénique), il importe de déterminer parmi eux, celui (ou ceux) vis-à-vis duquel (ou desquels) l'adaptation de l'agent pathogène sera la plus improbable. Au Centre I.N.R.A. d'Avignon, un tel travail est réalisé sur plusieurs couples hôte-parasite :

\section{Couple Tomate - Cladosporium fulvum}

Dans la perspective d'introduire la résistance à la cladosporiose dans les variétés de Tomate, les populations de C. fulvum, issues de feuilles collectées dans de nombreuses serres des différentes régions de production, sont inoculées systématiquement depuis 1977 à une gamme d'hôtes porteurs des différents gènes de résistance connus. Ce travail permet d'évaluer le niveau d'évolution des pathotypes et leur fréquence, donc de guider le choix des sélectionneurs (LATERrot et CleRJEAu, 1979).

\section{Couple Melon-Virus de la Mosaïque du concombre (CMV)}

Une étude menée de 1974 à 1978 par LEROux et al. (1979) dans la région d'Avignon a montré que parmi 1124 isolats de CMV prélevés sur hôtes sensibles, 35 p. 100 appartenaient au pathotype "Song ", agressif en inoculation mécanique sur le géniteur de résistance au CMV, PI 161375 , alors que ce géniteur n'avait jamais été cultivé dans la région.

Ce résultat montre que la résistance de Pl 161375 aux souches «communes " de CMV n'est pas suffisante pour assurer une protection satisfaisante du Melon vis-à-vis du CMV.

\section{Couple Poivron-Virus $Y$ de la Pomme de Terre (PVY)}

En effectuant sur différents géniteurs des repiquages successifs et accélérés à l'aide d'un isolat de PVY, POCHARD (1977) a pu obtenir des souches capables de surmonter certaines résistances au PVY, traduisant ainsi la relative fragilité de ces dernières.

Compte tenu du nombre très élevé de couples hôte-parasite existant dans le domaine des plantes légumières, la généralisation de ce type d'étude paraît bien difficile. Signalons, en outre, que ces travaux ont davantage pour but de comparer les limites d'efficacité de plusieurs gènes que de permettre l'élimination systématique des gènes faibles. En effet, de tels gènes, s'ils ne sont surmontés que par des pathotypes peu agressifs ou peu compétitifs, peuvent s'avérer utiles, surtout s'ils sont combinés à d'autres gènes. C'est ce que nous tenterons de démontrer.
2) Création de variétés possédant plusieurs mécanismes de résistance fondamentalement différents pour limiter les possibilités d'adaptation des agents pathogènes

La stabilité d'une résistance a d'autant plus de chances d'être grande que le nombre de mutations que l'agent pathogène doit réaliser pour s'y adapter, est élevé. Cette situation est obtenue lorsqu'un même génotype de plante cumule un maximum de mécanismes de résistance de nature fondamentalement différente. Pour y parvenir, deux voies semblent a priori adaptées: l'utilisation de résistances à déterminisme polygénique ou oligogénique et la combinaison, dans les variétés, de gènes de résistance conditionnant des mécanismes de défense différents. Examinons ces deux démarches.

\section{a) Utilisation de résistances oligogéniques ou polygéniques}

Dans beaucoup d'esprits, les résistances polygéniques sont synonymes de résistances horizontales ou non spécifiques, donc durables par définition. Cette assimilation parfois dogmatique nécessite des remarques. La première est que trop peu de résistances oligo ou polygéniques, introduites dans les variétés de plantes légumières, ont subi l'épreuve du temps, comparativement aux résistances monogéniques, pour que l'on puisse conclure sur leur meilleure aptitude à interdire l'adaptation des agents pathogènes.

Parmi les maladies que nous étudions, nous connaissons des exemples d'attaques sur des variétés possédant une résistance polygénique : souches "Song » de CMV agressives et pathogènes sur le géniteur de Melon PI 161375 , souches de Fusarium oxysporum f. sp. melonis provoquant le flétrissement des melons issus du géniteur «Kogane Nashi Makuwa", souches de Phytophthora capsici Leon. agressives sur certaines variétés de Poivron issues du géniteur PM 217.

Si l'on peut admettre que les résistances polygéniques reposent sur de nombreux gènes mineurs qui, individuellement, conditionnent des caractères de résistance peu efficaces mais qui, lorsqu'ils sont associés, confèrent aux plantes une résistance générale suffisante, on doit pouvoir en déduire que l'adaptation spécifique à quelques-uns de ces gènes peut être à l'origine d'une érosion de la résistance, suffisante pour la rendre inefficace. Cette hypothèse d'une adaptation de l'agent pathogène à certaines composantes de la résistance polygénique paraît vérifiée dans l'exemple du couple Piment- $P$. capsici (POCHARD et DAUBEZE, 1980) qu'il est bon de développer ici.

Exemple, chez Phytophthora capsici, d'une adaptation à diverses composantes d'une résistance polygénique, celle du géniteur PM 217 de Piment

Chez le Piment, la résistance à $P$. capsiciest étudiée grâce à une méthode d'inoculation sur section de tige décapitée qui permet de suivre, dans le temps, l'évolution des nécroses le long de la tige (POCHARD et CHAMBONNET, 1971). Avec les isolats les plus répandus, l'évolution de la vitesse de progression du champignon, chez la variété résistante Phyo 636 (issue de PM 217) comparativement à la variété sensible quasi isogénique Yolo Wonder, consiste en une décroissance rapide qui se stabilise progressivement à un niveau faible de palier entre le cinquième et le dixième jour suivant l'inoculation. Ce type de comportement n'est cependant pas général : avec certains isolats (souche 75) on assiste à partir du dixième jour, chez Phyo 636, à une reprise de croissance du champignon qui s'effectue à une vitesse 
progressivement comparable à celle observée chez Yolo Wonder. Avec d'autres isolats (souche 85 ), c'est la première phase de décroissance précoce qui n'intervient pas. Ces observations indiquent que la résistance de Phyo 636 repose sur deux types de mécanismes auxquels les souches de $P$. capsici peuvent être spécifiquement adaptées : un mécanisme A précoce et fugace et un mécanisme B d'action durable qui, apparemment, lui succède dans le temps (Pochard et DaUbeze, 1980).

D'autres types d'études réalisées par MoLot et al. (1977), sur les mêmes variétés, à différentes températures, montrent par ailleurs que, si la résistance de Phyo 636 est appréciable à l'égard de toutes les souches de $P$. capsici aux températures basses ou moyennes $\left(18^{\circ}, 22^{\circ} \mathrm{C}\right)$, il n'en est pas de même aux températures élevées : à $32^{\circ} \mathrm{C}$, certaines composantes de la résistance n'agissent plus et celles qui sont encore actives, donc responsables d'une moindre sensibilité, ne le sont pas à l'égard de toutes les souches. Ainsi, la souche 71 , adaptée aux mécanismes thermostables se montre-t-elle aussi pathogène sur Phyo 636 que sur Yolo Wonder à $32^{\circ} \mathrm{C}$.

Malgré de fortes présomptions, il n'a pas pu être confirmé que les composantes thermolabiles et thermostables de la résistance correspondent aux mécanismes $\mathrm{A}$ et $\mathrm{B}$ précédemment décrits.

\section{Conclusions pour le sélectionneur}

Si l'augmentation du nombre de composantes dans une résistance doit réduire les probabilités d'émergence de pathotypes adaptés à l'ensemble des mécanismes de défense de l'hôte, il importe que le sélectionneur disposant d'un géniteur intéressant de résistance polygénique, ne perde pas trop d'éléments de la résistance au cours de son programme. Ce risque est nécessairement élevé si le géniteur de départ est éloigné du type agronomique recherché et si aucune étude préalable n'a permis de définir les conditions d'inoculation ou de choix de souches qui soient susceptibles de trier les principales composantes de la résistance. Chez le Piment par exemple, les cas d'attaque au champ de variétés résistantes par $P$. capsici, concernent toujours des variétés possédant moins d'éléments de résistance que le géniteur de départ PM 217 (POCHARD et DAUBEZE, 1980).

Compte tenu des phénomènes évoqués, il paraît évident que la sélection récurrente est mieux adaptée au cumul optimum de gènes intéressants que les méthodes traditionnelles. Celles-ci peuvent cependant continuer de s'avérer très intéressantes pour additionner, dans les cultivars, des gènes connus induisant des mécanismes de défense de nature distincte.

\section{b) Combinaison de gènes majeurs de résistance monogé- nique}

Le cumul de plusieurs gènes majeurs connus agissant selon des processus différents peut être du plus grand intérêt. Le résultat peut être assimilé à une résistance oligogénique de synthèse de très haut niveau. Un exemple réussi de sélection, selon ce principe, est celui de la Tomate pour la résistance au virus de la Mosaïque du Tabac (TMV).

Il existe, en effet, deux types de résistance vis-à-vis des souches communes de TMV (LATERROT, 1973):

- une tolérance, contrôlée par le gène Tm-1,

- une hypersensibilité, contrôlée soit par Tm-2, soit par $\mathrm{Tm}-2^{2}$, qui sont allèles au même locus et indépendants de $\mathrm{Tm}-1$.
Ces gènes ne sont que partiellement dominants. A l'état homozygote, chacun d'eux entraîne une moindre fertilité qui, dans le cas de $\mathrm{Tm}-2^{2}$, peut être masquée, chez certains hybrides F1, par un effet d'hétérosis ou dans certaines conditions de culture. A l'état hétérozygote, ils ne peuvent être utilisés, du moins individuellement, contre les souches communes (race 0 ) : les plantes $\mathrm{Tm}-1 /$ + sont trop rapidement mosaïquées et les plantes $\mathrm{Tm}-2 /+$ ou $\mathrm{Tm} 2^{2} /+$ répondent à l'infection, en conditions de température et de luminosité élevées, par une hypersensibilité qui entraîne la nécrose partielle ou totale des plantes.

L'évidence de la différence qui existe entre les mécanismes de résistance contrôlés par ces divers gènes a pu être confirmée récemment par MOTOYOSHI et OSHIM A (1977) sur des protoplastes de mésophylle : sur cellules isolées, seul $\mathrm{Tm}-1$ peut réduire l'infection et la synthèse virale.

Ces différences de mode d'action entre gènes ont encouragé les sélectionneurs à créer des combinaisons hybrides permettant de cumuler les gènes. C'est le cas des hybrides français (LATERROT, 1977).

Alors que, dans la nature, on peut isoler assez fréquemment des souches adaptées à $\mathrm{Tm}-1$, à $\mathrm{Tm}-2$, ou à ces deux gènes simultanément (races 1,2 et 1-2 respectivement), on ne connaît pas de souches adaptées à Tm-2² (HALL, 1980). Cependant, on connaît des souches communes capables d'induire des nécroses systémiques sur les plantes homozygotes pour $\mathrm{Tm}-2^{2}$. Ces souches ne sont pas pathogènes vis-à-vis des plantes porteuses de Tm-1. Elles appartiennent au pathotype $0\left(\mathrm{~N} 2^{2}\right)$ (tabl. 1).

\section{TABLEAU 1}

Réactions contrôlées par chacun des 3 allèles de résistance connus chez la Tomate vis-à-vis des 5 pathotypes de TMV.

Reactions controlled by the 3 Tomato resistance genes to TMV, after contamination by the 5 known TMV pathotypes.

\begin{tabular}{cccccc}
\hline \hline \multirow{2}{*}{ Gènes de résistance } & \multicolumn{5}{c}{ Pathotypes de TMV } \\
\cline { 2 - 6 } & $\mathrm{O}$ & $\mathrm{O}\left(\mathrm{N} 2^{2}\right)$ & 1 & 2 & $1-2$ \\
\hline+ & $\mathrm{S}$ & $\mathrm{s}$ & $\mathrm{S}$ & $\mathrm{S}$ & $\mathrm{S}$ \\
$\mathrm{Tml}$ & $\mathrm{T}$ & $\mathrm{T}$ & $\mathrm{S}$ & $\mathrm{T}$ & $\mathrm{S}$ \\
$\mathrm{Tm}-2$ & $\mathrm{H}$ & $\mathrm{n}$ & $\mathrm{H}$ & $\mathrm{S}$ & $\mathrm{S}$ \\
$\mathrm{Tm}-2^{2}$ & $\mathrm{H}$ & $\mathrm{N}$ & $\mathrm{H}$ & $\mathrm{H}$ & $\mathrm{H}$
\end{tabular}

$\mathrm{S}=$ sensibilité, mosaïque ( $\mathrm{s}=$ sensibilité réduite, faible mosaïque) [susceptibility, mosaic ( $s=$ lower susceptibility, weak mosaic)] $\mathrm{T}=$ tolérance, réduction de la multiplication virale, pas de symptôme (tolerance, reduced virus multiplication, no symptom) $\mathrm{H}=$ hypersensibilité (hypersensitivity)

$\mathrm{N}=$ nécrose systémique $(\mathrm{n}=$ nécrose moins intense) [systemic necrosis $(n=$ less severe necrosis)]

Cet ensemble de données permet de comprendre l'intérêt des formules cumulant Tm-1 et Tm- $2^{2}$. Le seul défaut connu actuellement de cette combinaison est le risque de nécrose, par temps chaud, chez les plantes hétérozygotes pour Tm-1 et $\mathrm{Tm}-2^{2}$, consécutif à l'interaction d'un pathotype 1 . La situation est alors comparable à celle rencontrée chez une plante $\mathrm{Tm}-2^{2} /+$ contaminée par une souche commune (race 0 ). Pour cette raison, des accidents ont pu être observés chez des hybrides résistants cultivés à proximité de variétés sensibles prémunies contre le TMV quand la souche atténuée utilisée appartenant au pathotype 1 . Face à ce problème, une nouvelle souche prémunisante, peu ou pas nécrogène, a dû être recherchée. S'il se confirme qu'une 
telle souche a été trouvée, son utilisation lèvera la menace pesant sur le comportement des combinaisons génétiques cumulant $\mathrm{Tm}-1$ et $\mathrm{Tm}-2^{2}$.

\section{c) Combinaison de résistances monogéniques de haut niveau et de résistances polygéniques partielles}

Les inconvénients qui résultent d'une acquisition de virulence à l'égard des gènes majeurs de résistance absolue peuvent être considérablement réduits si on associe à ces gènes des gènes de résistance partielle. La sélection du Melon pour la résistance à la fusariose vasculaire et au CMV est actuellement conduite en ce sens.

Exemple de sélection pour la résistance à Fusarium oxysporum $f . s p$. melonis

La sélection du Melon, pour la résistance à la fusariose, a surtout fait appel, jusqu'à présent, aux gènes majeurs dominants Fom-1 et Fom-2 (MESSIAEN et al., 1962 ; RISSER, 1973). Ces gènes permettent de distinguer quatre races physiologiques $(0,1,2,1-2)$ présentes dans la nature.

Une résistance oligogénique récessive, provenant du géniteur «Kogane Nashi Makuwa », permet par ailleurs de contrôler toutes les souches peu agressives de $F$. oxysporum f. sp. melonis indépendamment de leur race. Cette résistance est, en revanche, surmontée par les isolats très agressifs.

Les études effectuées jusqu'à présent ont montré que les isolats appartenant au pathotype 1-2, donc pathogènes sur les variétés porteuses des deux gènes Fom-1 et Fom-2, sont peu agressifs. Le cumul, dans une même variété, de ces gènes et du système oligogénique récessif paraît donc la formule génétique la plus fiable pour assurer le contrôle de la fusariose.

\section{le Melon}

Exemple de sélection pour la résistance au $C M V$, chez

Lorsque la résistance au CMV s'exprime au champ chez un géniteur de Melon, elle se traduit par des symptômes de mosaïque plus faibles et plus tardifs que chez une variété sensible de type «Charentais » telle que Vedrantais. Il s'agit d'une résistance partielle qui résulte de l'association de deux mécanismes (tabl. 2):

— une résistance à l'inoculation mécanique, oligogénique récessive, qui se traduit par l'absence de symptômes en conditions d'infection contrôlées (RISSER et al.,1977). Cette résistance, présente chez les variétés P1 161375 et « Freeman's cucumber ", est inefficace vis-à-vis de certaines souches largement répandues dans la nature appelées souches « Song " (LEROUX et al., 1979).

- une résistance à la transmission du virus par le puceron vecteur Aphis gossypii Glov., à déterminisme monogénique dominant (PITRAT et LECOQ, 1980). Cette résistance présente dans les lignées PI 161375 et PI 414723, est aussi efficace vis-à-vis des souches "Song " que vis-àvis des souches «communes». Elle est cependant inopérante lorsque la transmission est assurée par d'autres pucerons vecteurs (Myzus persicae Sulz, Aphis fabae Scop.) (LECOQ et al., 1979, 1980).

Lorsque $A$. gossypii représente une fraction importante de la population de pucerons vecteurs dans une parcelle de Melon, le deuxième type de résistance peut être d'un très grand intérêt pratique. Il paraît cependant préférable de lui adjoindre la résistance oligogénique aux souches « communes ", c'est-à-dire d'utiliser toutes les composantes de la résistance de PI 161375, car seules les souches "Song" transmises par les pucerons autres que $A$. gossypii peuvent infecter un tel génotype.

\section{INTÉGRATION DE LA RÉSISTANCE VARIÉ- TALE AUX AUTRES MÉTHODES DE LUTTE}

Pour résumer l'historique des méthodologies et des concepts dans le domaine de la création de variétés résistantes chez les plantes légumières, rappelons que si, dans le passé, on a surtout exploité des résistances monogéniques de haut niveau, c'était pour des raisons que nous aurions mauvaise grâce à condamner aujourd'hui: l'absence de méthodes de lutte contre certaines maladies, d'une part. conférait à la création de variétés résistantes un caractère d'urgence; les excès de la lutte chimique, d'autre part, rendaient encore plus souhaitable que la lutte génétique lui soit substituée un jour ; or, aucune expérience précise ni aucune méthodologie éprouvée ne permettaient alors au sélectionneur d'envisager une autre alternative qu'une recherche et une utilisation intensive de résistances monogéniques.

\section{TABLEAU 2}

Réactions contrôlées par les mécanismes de résistance présents dans différentes variétés de Melon, vis-à-vis des souches de CMV, suivant le mode d'inoculation.

Reactions controlled by the resistance mechanisms effective in differential Muskmelon varieties, after contamination by 2 pathotypes inoculated mechanically or by aphids.

\begin{tabular}{|c|c|c|c|c|c|c|}
\hline \multirow{3}{*}{ Variétés } & \multicolumn{3}{|c|}{ Souche " commune" } & \multicolumn{3}{|c|}{ Souche "Song" } \\
\hline & \multirow{2}{*}{$\begin{array}{l}\text { Inoculation } \\
\text { mécanique }\end{array}$} & \multicolumn{2}{|c|}{ Inoculation par pucerons } & \multirow{2}{*}{$\begin{array}{l}\text { Inoculation } \\
\text { mécanique }\end{array}$} & \multicolumn{2}{|c|}{ Inoculation par pucerons } \\
\hline & & A. gossypii & $\begin{array}{c}\text { Autres } \\
\text { pucerons }\end{array}$ & & A. gossypii & $\begin{array}{c}\text { Autres } \\
\text { pucerons }\end{array}$ \\
\hline Vedrantais $\ldots \ldots \ldots \ldots \ldots \ldots . . . . . . . .$. & $S$ & S & $S$ & $\mathrm{~S}$ & $S$ & $S$ \\
\hline PI $414723 \ldots \ldots \ldots \ldots \ldots \ldots \ldots$ & $S$ & $\mathbf{R}$ & $\mathrm{S}$ & $S$ & $\mathrm{R}$ & $\mathrm{S}$ \\
\hline PI $161375 \ldots \ldots \ldots \ldots \ldots \ldots \ldots$ & $\mathbf{R}$ & $\mathrm{R}$ & $\mathbf{R}$ & $S$ & $\mathrm{R}$ & $S$ \\
\hline Freeman's cucumber .......... & $\mathbf{R}$ & $\mathrm{R}$ & $\mathrm{R}$ & $\mathrm{S}$ & $\mathrm{S}$ & $\mathrm{S}$ \\
\hline
\end{tabular}


Grâce à l'expérience et du fait de certains échecs, les objectifs de sélection ont évolué. L'essentiel aujourd hui, pour le sélectionneur, n'est plus, dans tous les cas, que la résistance assure à elle seule toute la protection de la plante mais est plutôt de faire valoir au mieux, toutes les sources de résistance qu'il a pu mettre en évidence, en faisant appel à d'autres méthodes de lutte. Les exemples qui suivent ont précisément pour but d'illustrer comment la résistance variétale peut devenir une composante nouvelle et importante de la lutte intégrée:

\section{1) Apport de la lutte chimique à la lutte génétique}

Amélioration de la durabilité des gènes "faibles" de résistance absolue (fig. 1).

Prenons le cas théorique d'une maladie épidémique évoluant selon une loi classique à intérêts composés vis-à-vis de laquelle on dispose, comme moyens de lutte, d'un fongicide systémique rémanent et d'une résistance variétale à haut risque de faillite. Si la récolte doit intervenir au bout d'un temps $\mathrm{T}$ supérieur à TF (rémanence du fongicide) ou à TR (temps nécessaire à l'apparition d'un pathotype surmontant la résistance), aucune des deux méthodes ne peut, isolément, assurer une protection satisfaisante, si ce n'est une répétition des traitements chimiques évidemment lourde d'inconvénients en culture légumière.

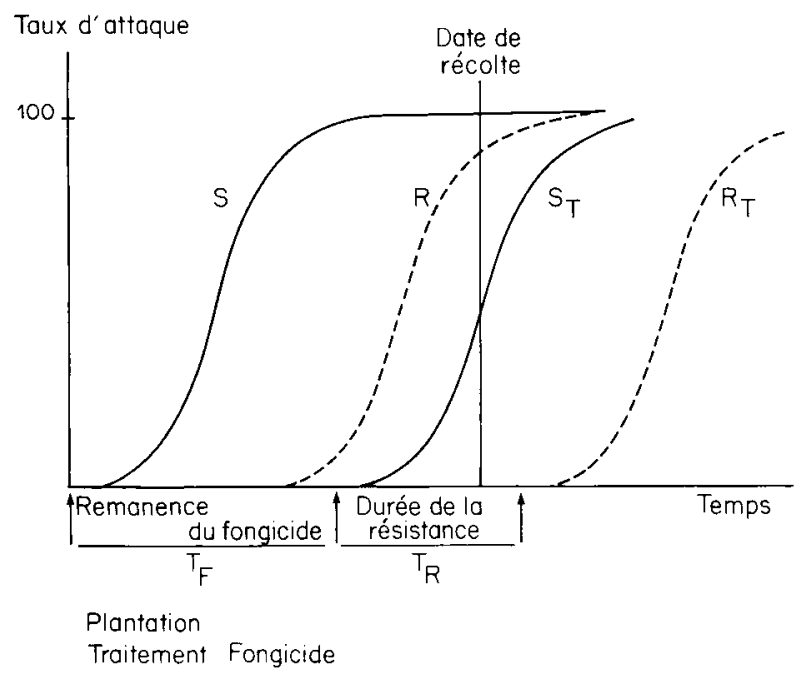

Figure 1

Courbes d'évolution théoriques d'une épidémie chez une variété sensible (-) et une variété résistante (- -) traitées (St et Rt) ou non traitées ( $S$ et $R$ ) à l'aide d'un fongicide rémanent.

Theorical epidemic evolution curves on a susceptible ( $\longrightarrow$ ) and a resistant (- - ) variety after a remanent fungicidal drench $(S t, R t)$ or without chemical protection $(S, R)$.

Si, en revanche, l'application du fongicide intervient précocement chez une variété résistante au lieu d'une variété sensible, le temps d'adaptation du parasite ne pourra pas être comptabilisé avant l'expiration de la fongistase liée au traitement. Aucune attaque ne pourra alors être observée avant un temps égal à TF + TR. Si ce temps est supérieur à $\mathrm{T}$ (ce qui est vraisemblable avec un fongicide rémanent et une plante maraîchère) la résistance doit demeurer absolue et stable tout au long de la culture. Elle permet alors de compléter l'action du fongicide à l'approche de la récolte et de limiter ainsi les résidus.
Il est vraisemblable que certaines maladies pourraient faire l'objet d'une telle stratégie de lutte, notamment $B$. lactucae sur la Laitue compte tenu de la nature des gènes exploités (JOHNSON et CRUTE, 1975) et de l'efficacité des nouveaux fongicides anti-Mildiou récemment mis au point (MARTIN, 1979).

Amélioration de la protection conférée par une résistance partielle grâce à des traitements fongicides réduits (fig. 2).

Les résultats que nous avons obtenus chez le Piment en comparant, chez une variété sensible à $P$. capsici (Yolo Wonder) et chez une variété partiellement résistante (Phyo 636), l'efficacité de plusieurs doses d'applications de phoséthyl-aluminium, nous paraissent très démonstratifs: Les plantes, élevées en pots, sous serre, ont été contaminées à l'âge de 2 mois, par apport d'inoculum au substrat, selon une méthode déjà décrite (CLERJEAU et BEYRIES, 1977). Nous avions choisi un isolat (souche 197) issu d'une variété résistante, très agressif sur "Phyo 636 " mais entraînant la mort de cette variété plus tardivement que chez "Yolo Wonder». Le fongicide a été appliqué au pied des plantes, le jour de la contamination, à différentes concentrations, à raison de $50 \mathrm{ml}$ de suspension par plante.

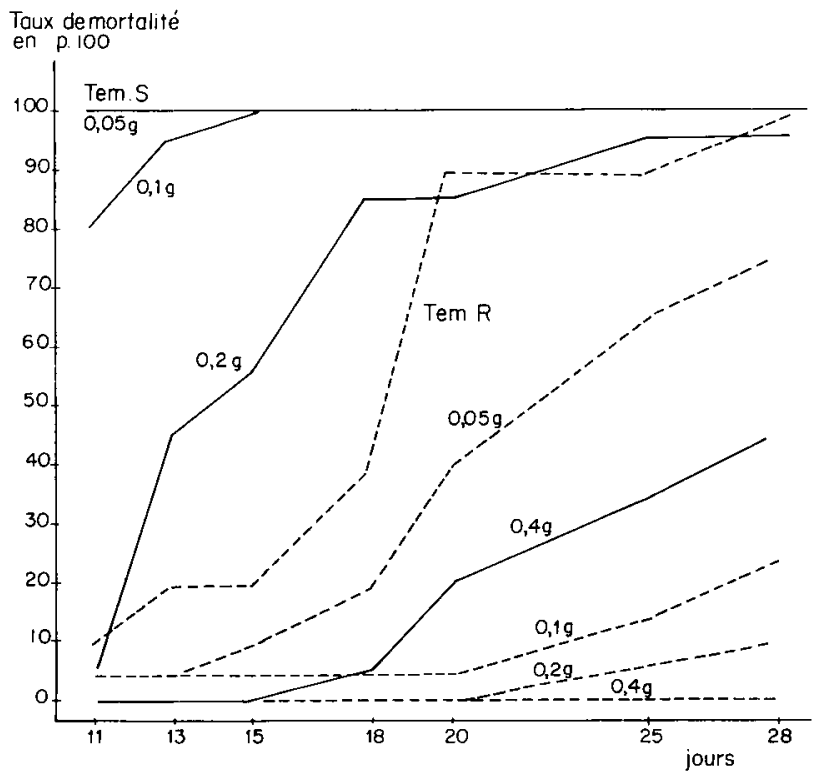

Figure 2

Evolution comparée des attaques de Phytophthora capsici sur des Poivrons de la variété sensible Yolo Wonder (-) et de la variété partiellement résistante Phyo 636 (--), traitées par des doses variables de phosethyl-aluminium $(100 \mathrm{ml}$ de bouillie par plante, contenant $0,05 \mathrm{~g}$ à $0,04 \mathrm{~g}$ de m.a.).

Compared evolution of attacks due to Phytophthora capsici on the susceptible (-) Yolo Wonder and the partially resistant (- .) Phyo 636 Pepper variety, after a drench with various doses of aluminium phosethyl $(100 \mathrm{ml}$ of mixture for each plant, containing $0,05 \mathrm{~g}$ to $0,4 \mathrm{~g}$ of a.m.).

L'évolution de la maladie observée chez les deux variétés, en fonction des différentes doses d'apport du fongicide, a permis de mettre en évidence, pour chacune des doses, une efficacité supérieure chez la variété résistante: Les courbes de la fig. 2 montrent en effet que, 1 mois après l'unique traitement, la presque totalité des plantes sensibles sont mortes aux doses inférieures ou égales à $0,2 \mathrm{~g}$ de $\mathrm{m}$. a. par plante et que 40 p. 100 sont mortes à la dose la plus forte, soit $0,4 \mathrm{~g}$. Chez les plantes résistantes, en revanche, 20 p. 100 seulement sont mortes à $0,1 \mathrm{~g}$ et 0 p. 100 à $0,4 \mathrm{~g}$. 
Ces résultats permettent, par conséquent, d'envisager une stratégie de traitements des variétés résistantes, confrontées à des souches très agressives, basées sur une utilisation de doses et d'une cadence d'application réduites.

\section{2) Apport de la lutte par procédés culturaux à la lutte génétique}

Comme les traitements chimiques, certaines interventions culturales susceptibles de réduire l'incidence des maladies peuvent contribuer à renforcer l'efficacité de résistances partielles. Un exemple particulièrement significatif est celui du désherbage des cultures de Melon pour lutter contre le CMV.

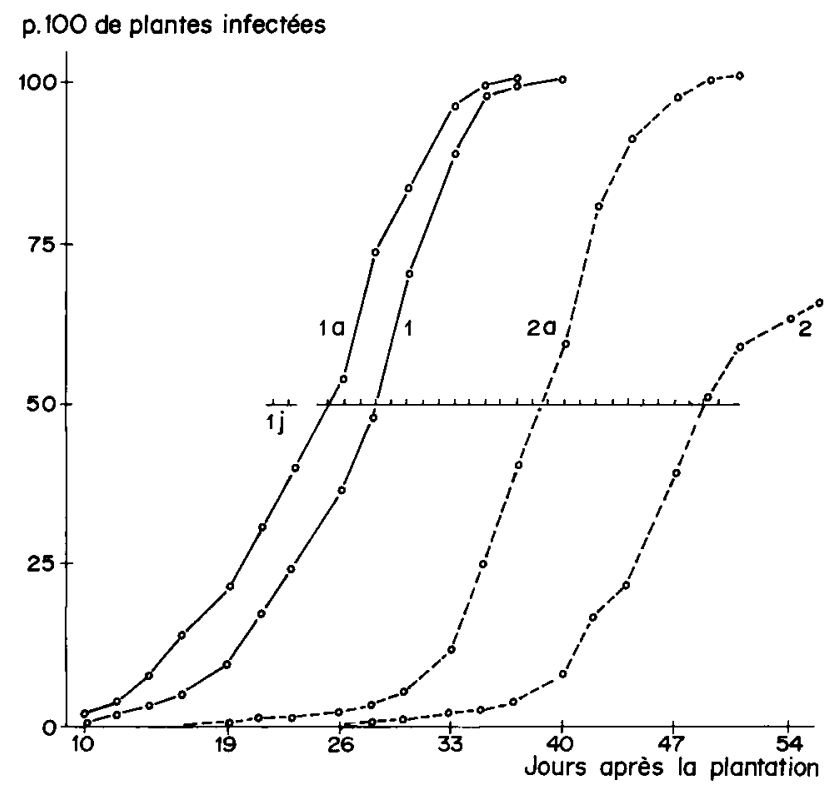

Figure 3

Influence $d u$ désherbage des bordures de parcelles sur le développement d'une épidémie de virus de la mosaïque du Concombre, dans des cultures de melons sensibles et résistants (Avignon, été 1979).

Influence of plots sides weeding on CMV epidemics in susceptible and resistant Muskmelon fields (Avignon, summer 1979).

\footnotetext{
Variété sensible Vedrantais (Susceptible variety Vedran tais) :

1:
1a: $\quad$ parcelle désherbée (weeded plot)
parcelle nósherbée (not weeded plot)

Variété résistante P1 161375 (Resistant variety P1 161 375)

2: $\quad$ parcelle désherbée (weeded plot)

2a: parcelle non désherbée (not weeded plot)
}

Les travaux de QUIOT et al. (1979) ayant montré que le désherbage des bordures des parcelles peut réduire sensiblement l'importance des sources de virus et de vecteurs aux abords des cultures, l'influence du désherbage sur la contamination des parcelles a été étudiée comparativement, au niveau d'une variété sensible ("Vedrantais") et au niveau d'une variété résistante ( $\mathrm{Pl} 161375 »)$, à la station de Pathologie végétale de Montfavet par LECOQ, PITRAT et LEROUX :

Lors de la campagne estivale 1979, caractérisée par une forte contamination des adventices par le CMV, le désherbage a retardé significativement le développement des épidémies tant au niveau de la variété sensible (ce qui confirme les résultats non publiés de QUIOT) que chez la variété résistante. Cependant, le retard a été plus marqué sur la seconde variété (fig. 3): par rapport aux parcelles correspondantes restées enherbées, le retard a été de 3 jours pour atteindre le taux de $50 \mathrm{p} .100$ de maladie chez «Vedrantais », et environ 10 jours chez «Pl 161375 ». De ce fait, le retard dans le développement de l'épidémie (toujours apprécié au taux de maladie 50 p. 100) qui était de 14 jours dans la parcelle de variété résistante par rapport à la parcelle de variété sensible, en absence de désherbage, est passé à 24 jours lorsqu'à la résistance variétale on a combiné la pratique du désherbage.

Cet effet favorable du désherbage sur l'efficacité de la résistance n'a pas été observé lors de la campagne de printemps de 1979. Les sources de virus étant alors plus rares parmi les mauvaises herbes, le rôle du désherbage ne pouvait être que limité.

Ces résultats ont conduit les auteurs à expérimenter d'autres pratiques culturales pour définir les conditions agronomiques dans lesquelles la résistance génétique partielle du Melon au CMV peut avoir une expression optimale (paillage plastique, pulvérisation d'huiles, etc.). Ces travaux sont en cours.

\section{CONCLUSION}

La notion essentielle à dégager de l'examen de l'évolution de la création de variétés résistantes chez les plantes légumières nous paraît être la diversification des stratégies de sélection: Alors que, dans le passé, seules les résistances de haut niveau, induites par des gènes majeurs, étaient retenues, aujourd'hui, le sélectionneur étudie l'intérêt de toutes les sources de résistance dont il dispose quel que soit leur support génétique. Les critères de choix ont, en effet, évolué. L'un des principaux est la durabilité des gènes. L'amélioration de la durabilité qui repose généralement sur le cumul de plusieurs systèmes géniques peut amener le sélectionneur à retenir des gènes n'assurant qu'une résistance partielle. Des études menées en collaboration avec des phytopathologistes sont alors nécessaires pour préciser les conditions phytotechniques dans lesquelles on peut espérer une expression optimale de la résistance. Nous assistons donc progressivement à une intégration de plus en plus poussée de la résistance variétale aux autres méthodes de lutte.

Cette évolution dans les conceptions ne manquera pas de poser des problèmes de formation et d'information à l'égard des praticiens maraîchers ou de leurs techniciens. Pour ces derniers, en effet, la résistance des plantes est encore assimilée à une immunité. Il conviendra donc de leur expliquer nos choix, en particulier pourquoi, dans certains cas, une moindre sensibilité sera préférable à une immunité et comment certaines plantes moins sensibles peuvent devenir résistantes dans des conditions de cultures particulières.

I] est important d'ajouter que si le secteur de la création variétale évolue en prenant en compte l'intérêt des autres méthodes de lutte contre les maladies, à l'inverse, les phytotechniciens et phytopathologistes doivent raisonner en fonction des gènes de résistance présents chez les plantes. L'exemple de la prémunition de la Tomate contre le TMV 
montre, en effet, qu'en fonction de l'appartenance raciale de la souche atténuée choisie, la prémunition peut être, à côté de la résistance variétale, un atout supplémentaire dans la lutte, ou bien une contre-indication à l'usage de la résistance variétale dans certaines conditions.

Toutes ces observations montrent, en fait, que l'évolution des orientations de la sélection variétale pour la résistance aux maladies est parallèle à l'évolution des collaborations entre les généticiens sélectionneurs et les phytopathologistes.

Reçu le 18 septembre 1980 Accepté le 16 octobre 1980.

\section{RÉFÉRENCES BIBLIOGRAPHIQUES}

Clerjeau M., Beyries A., 1977. Etude comparée de l'action préventive et du pouvoir systémique de quelques fongicides nouveaux (phosphites, prothiocarbe, pyroxychlore) sur Poivron, vis-à-vis de Phytophthora capsici. Phytiatr. Phytopharm., 26, 73-84.

Clerjeau M., Laterrot H., Pitrat M., 1979. Création de variétés résistantes aux maladies chez les plantes maraîchères. Bull. tech. inf., 337, 101-114

Hall T. J., 1980. Resistance at the Tm-2 locus in the Tomato mosaic virus. Euphytica, 29, 189-197.

Johnson G., Crute I., 1975. Bremia and the way it affects lettuce varieties wich are resistant. The Grower. Oct. 25, 742-744.

Laterrot H., 1973. Résistance de la Tomate au virus de la mosaique du Tabac. Difficultés rencontrées pour la sélection de variétés résistantes. Ann. Amélior. Plantes, 23, (4), 287-313.

Laterrot H., 1977. Résistance de la Tomate au virus de la mosaïque du Tabac. Etat actuel de la sélection. P.H.M., 175, 13-20.

Laterrot H., Clerjeau M., 1979. Détermination des pathotypes de Fulvia fulva (= Cladosporium fulvum) présents sur Tomate dans les serres françaises. Ann. Amélior. Plantes, 29, (4), 447-462.

Lecoq H., Cohen S., Pitrat M., Labonne G., 1979. Resistance to Cucumber Mosaic Virus transmission by Aphids in Cucumis melo. Phytopathology, 69, 1223-1225.

Lecoq H., Labonne G., Pitrat M., 1980. Specificity of resistance to virustransmission by aphids in Cucumis melo. Ann. Phytopathol. 12, (2), 139-144.

Leroux J. P., Quiot J. B., Lecoq H., Pitrat M., 1979, Mise en évidence et répartition dans le Sud-Est de la France d'un pathotype particulier de virus de la mosaïque du Concombre. Ann. Phytopathol., 11, (3), 431-438.

Martin C., 1979. Essai de fongicides systémiques anti-Bremia par incorporation dans le terreau. Rapp. activité S.C.R.E.A.P.O., 19, av. de Grande Bretagne, 66025 - Perpignan.

Messiaen C. M., Risser G., Pecaut P., 1962. Etude des plantes résistantes au Fusarium oxysporum $\mathrm{f}$. sp. melonis dans la variété de Melon "Cantaloup Charentais». Ann. Amélior. Plantes, 12, (1), 157-164
Molot P. M., Clerjeau M., Mas P., Ricci P., 1977. Rôle du capsidiol dans la résistance génétique du Piment (Capsicum annuum) au Phytophthora capsici. "Capsicum 77 » C.R. 3e Cong. Eucarpia 5-8 juil. Edit. E. Pochard (I.N.R.A., 84140 - Montfavet-Avignon France), $137-146$

Motoyoshi F., Oshima N., 1977. Expression of genetical controlled resistance to Tobacco mosaic virus infection in isolated tomato leaf mesophyl protoplasts. J. Gen. Virol., 34, 499-506

Pitrat M., Lecoq H., 1980. Inheritance of resistance to cucumber mosaic virus transmission by Aphis gossypii in Cucumis melo. Phytopathology, 70, 958-961.

Pochard E., 1977. Etude de la résistance aux souches européennes de virus $Y$ de la Pomme de terre (PVY) chez le Piment. "Capsicum 77 ", C.R. $3^{e}$ Congrès Eucarpia, 5-8 juil. Edit. E. Pochard (I.N.R.A. 84140 Montfavet-Avignon), France, 109-118.

Pochard E., Chambonnet D., 1971. Méthodes de sélection du Piment pour la résistance au Phytophthora capsici et au virus du Concombre. Eucarpia meeting on Capsicum, Torino, 1971. Ann. Fac. Sci. Agr., Univ. Torino, 7, 270-281.

Pochard E., Daubeze A. M., 1980. Recherche et évaluation des composantes d'une résistance polygénique: La résistance du Piment à Phytophthora capsici. Ann. Amélior. Plantes, 30 (4), 359-379

Quiot J. B., Marchoux G., Douine L., Vigouroux A., 1979. Ecologie et épidémiologie du virus de la mosaïque du Concombre dans le Sud-Est de la France. V. Rôle des espèces spontanées dans la conservation du virus. Ann. Phytopathol., 11 (2), 325-348.

Risser G., 1973. Etude de l'hérédité de la résistance du Melon (Cucumis melo) aux races 1 et 2 de Fusarium oxysporum f.sp. melonis. Ann. Amélior. Plantes, 23 (1), 259-263.

Risser G., Pitrat M., Rode J. C., 1977. Etude de la résistance du Melon (Cucumis melo L.) au virus de la mosaïque du Concombre. Ann. Amélior. Plantes, 27, 509-522.

Van Der Plank J. E., 1968. Disease resistance in plants. Academic Press Inc, New York and London, $206 \mathrm{p}$. 